\title{
SENSITIVITY ANALYSIS TO EVALUATE THE TRANSPORT PROPERTIES OF CdZnTe DETECTORS USING ALPHA PARTICLES AND LOW-ENERGY GAMMA-RAYS
}

\author{
KYUNG-O KIM ${ }^{1}$, WOO SANG AHN ${ }^{2}$, TAE-JE KWON ${ }^{1}$, SOON YOUNG KIM ${ }^{3}$, JONG KYUNG KIM ${ }^{1, *}$, and \\ JANG-HO HA ${ }^{4}$ \\ ${ }^{1}$ Department of Nuclear Engineering, Hanyang University \\ Seoul 133-791, Korea \\ ${ }^{2}$ Department of Radiation Oncology, Asan Medical Center \\ Seoul 138-736, Korea \\ ${ }^{3}$ RADCORE Co., Ltd \\ Daejeon 305-509, Korea \\ ${ }^{4}$ Advanced Radiation Detection Instrument \& Sensor Lab, Korea Atomic Energy Research Institute \\ Daejeon 305-353, Korea \\ ${ }^{*}$ Corresponding author. E-mail : jkkim1@ @ hanyang.ac.kr
}

Received September 09, 2010

Accepted for Publication June 01, 2011

A sensitivity analysis of the methods used to evaluate the transport properties of a CdZnTe detector was performed using two different radiations ( $\alpha$ particle and gamma-ray) emitted from an ${ }^{241} \mathrm{Am}$ source. The mobility-lifetime products of the electron-hole pair in a planar CZT detector $\left(5 \times 5 \times 2 \mathrm{~mm}^{3}\right)$ were determined by fitting the peak position as a function of biased voltage data to the Hecht equation. To verify the accuracy of these products derived from $\alpha$ particles and low-energy gamma-rays, an energy spectrum considering the transport property of the CZT detector was simulated through a combination of the deposited energy and the charge collection efficiency at a specific position. It was found that the shaping time of the amplifier module significantly affects the determination of the $(\mu \tau)$ products; the $\alpha$ particle method was stabilized with an increase in the shaping time and was less sensitive to this change compared to when the gamma-ray method was used. In the case of the simulated energy spectrum with transport properties evaluated by the $\alpha$ particle method, the peak position and tail were slightly different from the measured result, whereas the energy spectrum derived from the low-energy gamma-ray was in good agreement with the experimental results. From these results, it was confirmed that low-energy gamma-rays are more useful when seeking to obtain the transport properties of carriers than $\alpha$ particles because the methods that use gamma-rays are less influenced by the surface condition of the CZT detector. Furthermore, the analysis system employed in this study, which was configured by a combination of Monte Carlo simulation and the Hecht model, is expected to be highly applicable to the study of the characteristics of CZT detectors.

KEYWORDS : Semiconductor Detector, CdZnTe, Hecht Equation, Charge Collection Efficiency, Tail Effect

\section{INTRODUCTION}

The Cadmium Zinc Telluride (CZT) detector is promising for radiation detection and medical imaging owing to its excellent energy resolution, sufficient bandgap energy, and room temperature operation ${ }^{[1-2]}$. Regardless of these advantages, it is known that the CZT detector

${ }^{*}$ Corresponding Author

Tel: +82-2-2220-0464, Fax: +82-2-2294-4800

E-mail: jkkim1@ hanyang.ac.kr has poorer transport properties than other semiconductor detectors, signifying that electron-hole pairs when they are generated cannot be completely collected on each electrode (i.e., the cathode and the anode) ${ }^{[3-4]}$. This phenomenon leads to a significant distortion of the measured energy spectrum. Hence, the mobility-lifetime products of the electron-hole pair $\left((\mu \tau)_{\mathrm{e}}\right.$ and $\left.(\mu \tau)_{\mathrm{h}}\right)$ are often considered in accurate evaluations of the characteristics of a CZT detector.

A common method to determine the transport properties of these types of detector is based on their responses to $\alpha$ particles ${ }^{[5-7]}$. This method, while known to 
be very sensitive to experimental conditions such as the shaping time and the measuring temperature, is an effective tool for studying the mobility-lifetime products of carriers due to the short mean free path of the $\alpha$ particle. Table 1 shows previous evaluations of the transport properties of commercial CZT detectors based on a typical evaluation method ${ }^{[8-11]}$. It was found that previously published measured values show considerable differences, in fact nearly a 10-fold difference, between the maximum and minimum values for each $(\mu \tau)$ product.

In this study, the simultaneous measurement of two different radiations, $\alpha$ particles and gamma-rays, was performed to analyze the extent of the sensitivity of the $\alpha$ particle method to the shaping time of an amplifier module and to confirm the possible application of low-energy gamma-rays as an alternative to the common method. The measured results were also compared with the energy spectrum simulated by MCNPX code ${ }^{[12]}$ to confirm the accuracy of the experimental values.

\section{METHODS AND MATERIALS}

To determine the mobility-lifetime products of an electron-hole pair in a semiconductor detector, measured energy spectrums under various bias voltages are required and specific variation of these spectrums should be analyzed by a suitable model matching each detector's type (i.e., planar, pixel, strip, and others). Herein are specific descriptions of methods used in the experiments and simulations.

\subsection{The Mobility-lifetime Determination}

A commercial-grade planar CZT detector $(5 \times 5 \times 2$ $\mathrm{mm}^{3}$ ) manufactured by eV Products was utilized to analyze the charge transport properties. The CZT crystal in this detector was grown by the High-Pressure Bridgman (HPB) method ${ }^{[13]}$, and the two electrodes that collected the charge

Table 1. Transport Properties of CZT Detectors Evaluated by Various Authors (using the alpha particle)

\begin{tabular}{c|c|c}
\hline Author & $\begin{array}{c}(\mu \tau)_{\mathrm{e}} \\
{\left[\mathrm{cm}^{2} / \mathrm{V}\right]}\end{array}$ & $\begin{array}{c}(\mu \tau)_{\mathrm{h}} \\
{\left[\mathrm{cm}^{2} / \mathrm{V}\right]}\end{array}$ \\
\hline Y. Nemirovsky & $4.0 \times 10^{-4}$ & $8.0 \times 10^{-5}$ \\
\hline J. E. Toney & $6.0 \times 10^{-3}$ & $3.0 \times 10^{-4}$ \\
\hline M. C. Veale & $1.25 \times 10^{-3}$ & - \\
\hline S. H. Park & $1.69 \times 10^{-3}$ & $3.76 \times 10^{-5}$ \\
\hline
\end{tabular}

${ }^{*}(\mu \tau)_{e}$ and $(\mu \tau)_{h}$ products were evaluated using an alpha particle and gamma-ray, respectively carriers were made of $10 \mu \mathrm{m}$-thick platinum. For the determination of the mobility-lifetime products for the electron and hole, the selected detector was irradiated with low-energy gamma-rays $\left(\mathrm{E}_{\gamma}<60 \mathrm{keV}\right)$, and $5.5 \mathrm{MeV}$ $\alpha$ particles were emitted from an ${ }^{241} \mathrm{Am}$ isotope through the front surface of the detector. The signals from the CZT detector were processed by a preamplifier and an amplifier (ORTEC 590A), of which the shaping time was changed from a set minimum time $(0.5 \mu \mathrm{s})$ to a set maximum time ( $3 \mu s)$. The energy spectrum was obtained from a MultiChannel Analyzer (ORTEC 919E), and these experiments were performed at room temperature. Figure 1 shows the CZT detector and a circuit board surrounded with aluminum housing which was designed to prevent external noise. The series of the experiments performed in this study progressed as the bias voltage was changed from $-200 \mathrm{~V}$ to $200 \mathrm{~V}$.

\subsection{Energy Spectrum Simulation Considering the Transport Properties of CZT Detectors}

The Hecht equation optimized for a planar CZT detector was employed to derive the charge collection efficiency, as a function of the CZT depth, and the mobility-lifetime products ${ }^{[14]}$. This equation allows for the calculation of the charge collection efficiency, which here is represented as the ratio of the number of charge carriers induced at the electrodes to the total number of carriers created by the radiation interaction (see Eq. (1)). If most interactions between the radiation emitted from the source and semiconductor material occur on the near-incident surface, this equation can also be modified to the simplest form to extract the mobility-lifetime from the peak variation of measured spectrums, as shown in Eq. (2) ${ }^{[5]}$. Because the mean free paths of the gamma-ray and $\alpha$ particles used in this experiment were less than $200 \mu \mathrm{m}$ and were a few

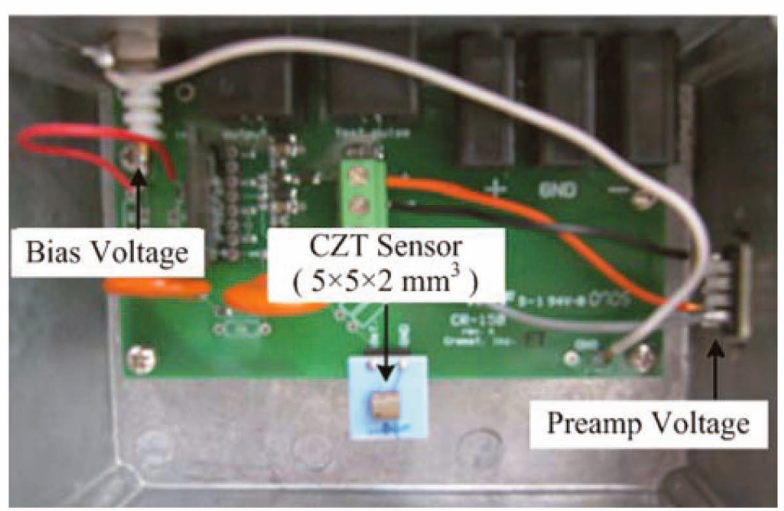

Fig. 1. Experiment Apparatuses (i.e., CZT Detector, Circuit Board, and Aluminum Housing) Used for Measuring the Transport Properties of an Electron-hole Pair in a Planar CZT Detector 
$\mu \mathrm{m}$ in the CZT crystal, respectively, these equations can be adopted for an analysis of the transport property of CZT detector, as follows:

$$
\begin{aligned}
& \eta(z)=\frac{(\mu \tau)_{e} \cdot E}{D}\left[1-e^{-\frac{(D-z)}{(\mu \tau)_{e} \cdot E}}\right]+\frac{(\mu \tau)_{h} \cdot E}{D}\left[1-e^{-\frac{z}{(\mu \tau)_{h} \cdot E}}\right] \\
& \left\{\begin{array}{l}
\eta(z)=\frac{(\mu \tau)_{e} \cdot E}{D}\left[1-e^{-D^{D}(\mu)_{e} \cdot E}\right] \\
\eta(z)=\frac{(\mu \tau)_{h} \cdot E}{D}\left[1-e^{-\frac{D}{(\mu \tau)_{h} \cdot E}}\right]
\end{array}\right\}
\end{aligned}
$$

Here, $\eta$ is the charge collection efficiency, $D$ is the detector thickness, and $(\mu \tau)_{\mathrm{e}}$ and $(\mu \tau)_{\mathrm{h}}$ are the mobilitylifetime products for the electron and hole, respectively. $\mathrm{E}$ is the strength of the electric field in the CZT sensor.

The brief flow chart in Figure 2 was designed to simulate the energy spectrum of the incident radiation considered the transport property of the CZT detector. The mobility-lifetime products were obtained by the above-mentioned method, and the deposited energy along the interaction position of the incident radiation was calculated using a DBCN card ${ }^{[12]}$, which is used primarily for debugging problems in the code itself, in this case the MCNPX code. In the calculation, the radiation source (26.3 keV and $59.5 \mathrm{keV}$ gamma rays) was assumed to be perpendicularly incident on the planar detector from ${ }^{241} \mathrm{Am}$; it was located $3 \mathrm{~cm}$ away from the negative contact to simulate the actual condition identically. The collected charge on each electrode was also investigated by a combination of the deposited energy and the charge collection efficiency at a specific position, and the energy spectrum was obtained through the accumulation of the energy that collected on the electrodes. The symmetrical peak broadening caused from the electronic noise (including the leakage current) was approximated with the Gaussian shape, which was estimated from the experimental results ${ }^{[11]}$. Finally, the energy spectrum obtained from this procedure was compared with the experimental results to verify the accuracy of the derived mobility-lifetime products.

\section{RESULTS AND DISCUSSIONS}

Table 2 shows the $(\mu \tau)_{\mathrm{e}}$ and $(\mu \tau)_{\mathrm{h}}$ products determined by two types of radiation emitted from the ${ }^{241} \mathrm{Am}$ isotope as the shaping time of the amplifier module was changed from $0.5 \mu$ s to $3 \mu \mathrm{s}$. It was found that an evaluated values for the $(\mu \tau)_{\mathrm{h}}$ product could be increased steadily by increasing the shaping time, regardless of the type of radiation used. In contrast, this trend in the $(\mu \tau)_{\mathrm{e}}$ product differed slightly from the trend noted with the incident radiation. Specifically, the $(\mu \tau)_{\mathrm{e}}$ product derived from the $\alpha$ particles was more stable than that from the low-energy
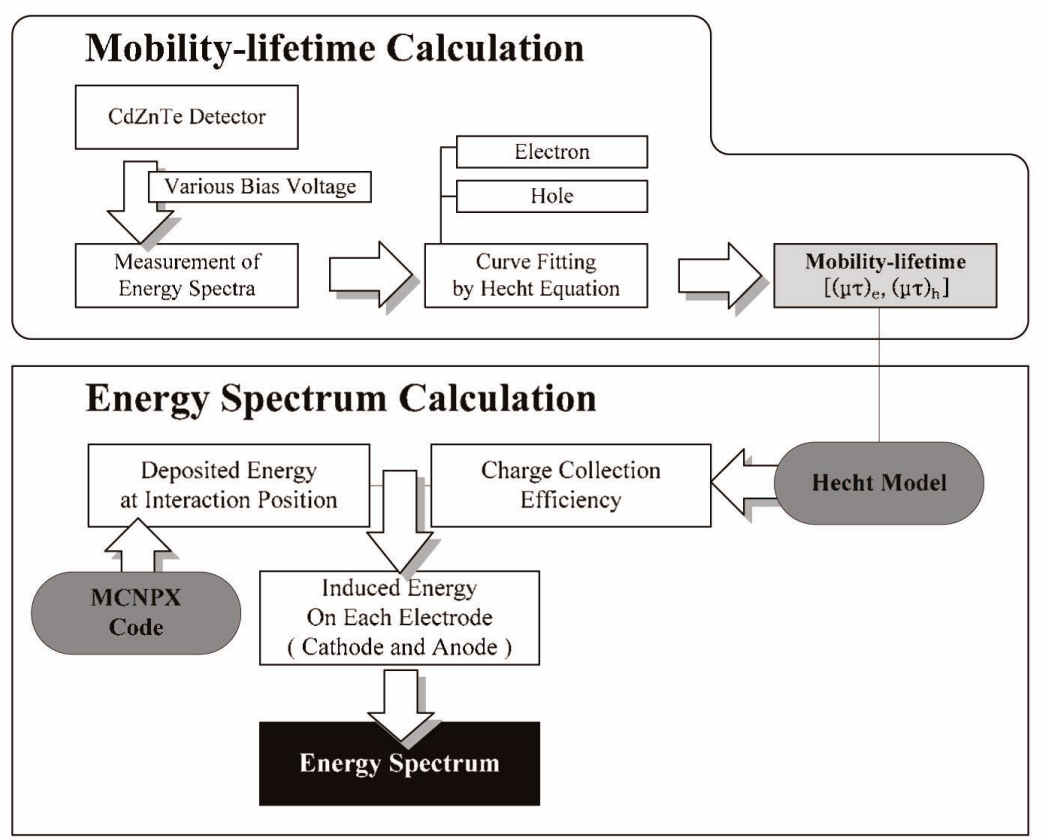

Fig. 2. A Brief Flow Chart of the Simulation of the Energy Spectrum Considered with the Transport Property of the CZT Detector 
gamma-rays during changes in the shaping time (see each standard deviation in Table 2). The ratio of the two mobility-lifetime products $\left((\mu \tau)_{\mathrm{e}} /(\mu \tau)_{\mathrm{h}}\right)$ decreased rapidly with an increase in the shaping time up to approximately half of that obtained at the shortest shaping time. From these results, it was confirmed that the shaping time of the amplifier module affected the evaluation for the $(\mu \tau)$ products. It was also found that the method involving the use of low-energy gamma-ray was more sensitive to this change compared to the $\alpha$ particle method.
Figure 3 shows the optimized cases among the charge collection efficiencies as a function of the bias voltage used to determine the $(\mu \tau)$ products. In the case of the method using low-energy gamma-rays, the experimental results at a shaping time of $1.5 \mu \mathrm{s}$ were the most stable, perfectly matching the fitting graph generated by the Hecht equation. On the other hand, those from $\alpha$ particle method were gradually stabilized by increasing the shaping time of the amplifier module. However, it was considered that the $(\mu \tau)_{\mathrm{e}}$ product determined from the optimized condition

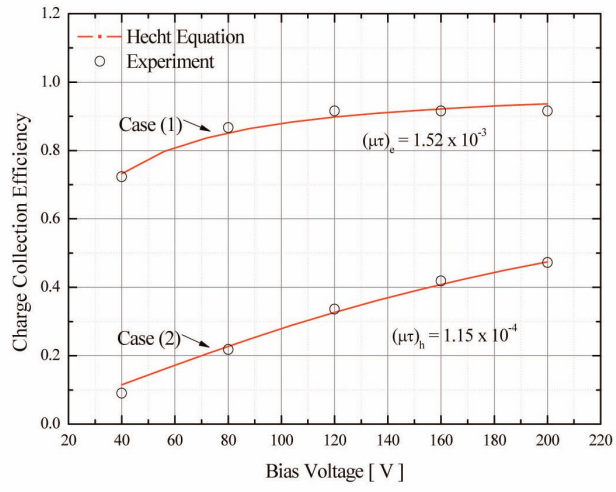

(a) Gamma-ray Method (Shaping Time $=1.5 \mu \mathrm{s}$ )

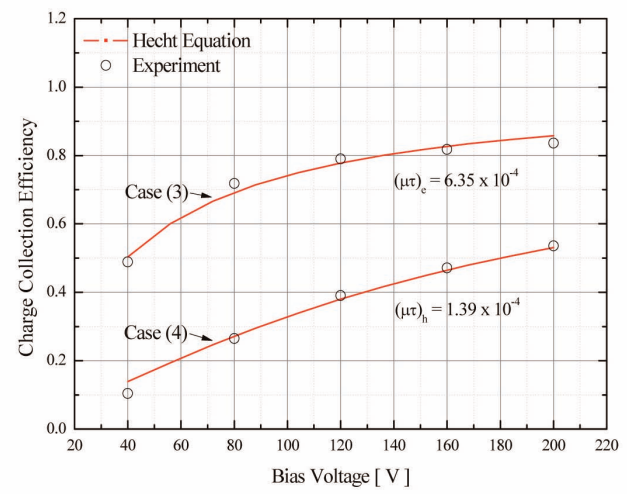

(b) Alpha Particle Method (Shaping Time $=3.0 \mu \mathrm{s}$ )

Fig. 3. Charge Collection Efficiency as a Function of the Bias Voltage for the Gamma-ray and Alpha Particle Methods

Table 2. Mobility-lifetime Products of an Electron-hole Pairs Derived from Gamma-rays and Alpha Particles at Various Shaping Times

\begin{tabular}{|c|c|c|c|c|}
\hline Used Radiation & $\begin{array}{c}\text { Shaping Time } \\
{[\mu \mathrm{s}]}\end{array}$ & $\begin{array}{c}(\mu \tau)_{\mathrm{e}} \\
{\left[\mathrm{cm}^{2} / \mathrm{V}\right]}\end{array}$ & $\begin{array}{c}(\mu \tau)_{\mathrm{h}} \\
{\left[\mathrm{cm}^{2} / \mathrm{V}\right]}\end{array}$ & $\frac{(\mu \tau)^{e}}{(\mu \tau)_{h}}$ \\
\hline \multirow{3}{*}{$\gamma$-ray } & 0.5 & $1.36 \times 10^{-3}$ & $6.66 \times 10^{-5}$ & 20.42 \\
\hline & 1.5 & $1.52 \times 10^{-3}($ Case 1$)$ & $1.15 \times 10^{-4}($ Case 2$)$ & 13.22 \\
\hline & 3.0 & $2.23 \times 10^{-3}$ & $2.03 \times 10^{-4}$ & 10.99 \\
\hline \multicolumn{2}{|c|}{ Standard Deviation $[\sigma]$} & $3.78 \times 10^{-4}$ & $5.65 \times 10^{-5}$ & - \\
\hline Used Radiation & $\begin{array}{c}\text { Shaping Time } \\
{[\mu \mathrm{s}]}\end{array}$ & $\begin{array}{c}(\mu \tau)_{\mathrm{e}} \\
{\left[\mathrm{cm}^{2} / \mathrm{V}\right]}\end{array}$ & $\begin{array}{c}(\mu \tau)_{\mathrm{h}} \\
{\left[\mathrm{cm}^{2} / \mathrm{V}\right]}\end{array}$ & ${\frac{(\mu \tau)^{e}}{(\mu \tau)_{h}}}$ \\
\hline \multirow{3}{*}{$\alpha$ particle } & 0.5 & $5.40 \times 10^{-4}$ & $6.16 \times 10^{-5}$ & 8.77 \\
\hline & 1.5 & $7.15 \times 10^{-4}$ & $1.12 \times 10^{-4}$ & 6.38 \\
\hline & 3.0 & $6.35 \times 10^{-4}($ Case 3$)$ & $1.39 \times 10^{-4}($ Case 4$)$ & 4.57 \\
\hline \multicolumn{2}{|c|}{ Standard Deviation $[\sigma]$} & $7.15 \times 10^{-5}$ & $3.21 \times 10^{-5}$ & - \\
\hline
\end{tabular}


of the $\alpha$ particle method (Case 3) was about two times less than the previously known value for a commercial CZT detector ${ }^{[15-16]}$. Therefore, the energy spectrum of some of the radiation emitted from the ${ }^{241} \mathrm{Am}$ source was simulated while taking into account the transport properties for the electron-hole pairs (from Case 1 to Case 4). This was then compared with the experimental results in an effort to evaluate the accuracy of the determined products.

Figure 4 shows the energy spectrum derived from the methods using the low-energy gamma-rays and the $\alpha$ particles. The energy spectrum derived from the gammaray method was in excellent agreement with the experimental results in the energy range from $0-70 \mathrm{keV}$. In another case, the peak position (region 1) and the tail (region 2) in the spectrum differed slightly from those of the measured result. It was expected that the $(\mu \tau)_{\mathrm{e}}$ product derived from the $\alpha$ particle method would be relatively low given that the electron-hole pairs generated from the $\alpha$ particle (mean free path: $\sim$ a few $\mu \mathrm{m}$ ) were influenced by the distortion of the electric field at the incident

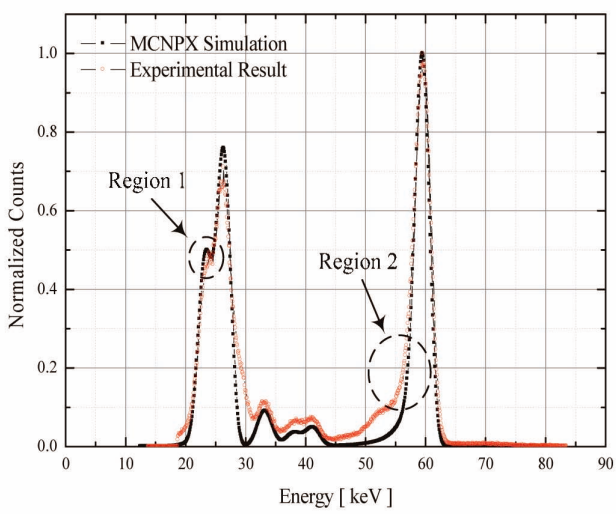

(a) Gamma-ray Method

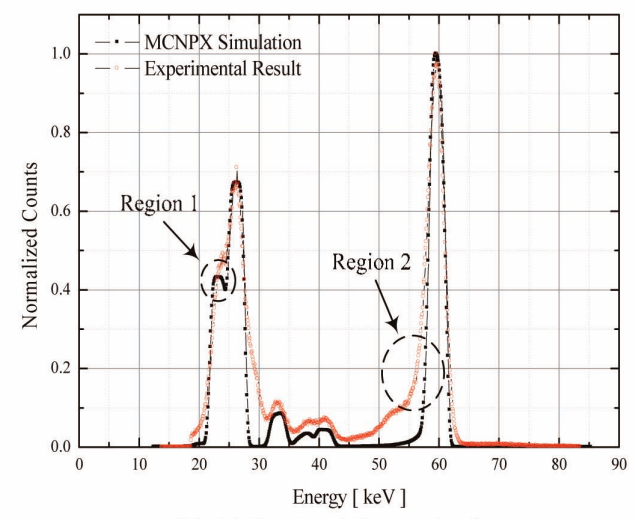

(b) Alpha Particle Method

Fig. 4. A Comparison of Experimental and Simulated Results considering the Transport Properties of the Electronhole Pair surface ${ }^{[17]}$. This indicates that the method involving the use of low-energy gamma-rays is useful for investigating the transport properties of semiconductor detectors.

\section{CONCLUSIONS}

The simultaneous measurement of two different types of radiation emitted from an ${ }^{241} \mathrm{Am}$ isotope was performed in an effort to investigate the sensitivity of the $\alpha$ particle method to the shaping time of the amplifier module as well as the efficiency of the method using low-energy gamma-rays. The energy spectrum considering the transport properties of the CZT detector was also simulated to compare the accuracy of derived values to the experimental results. It was confirmed that the $\alpha$ particle method resulted in steadily stabilization with an increase in the shaping time of the amplifier module. This method was also less sensitive to this change than the gamma-ray method. While energy spectrum derived from the $\alpha$ particle method showed some degree of discrepancy (i.e., the peak position and tail) from the experimental results, the energy spectrum derived from this method using low-energy gamma-rays showed good agreement with the experimental results over the entire energy range from $0-70 \mathrm{keV}$. Therefore, it is considered that low-energy gamma-rays are more useful when seeking to obtain the transport properties of carriers than $\alpha$ particles because the method involving the use of gamma-rays is influenced less by the surface condition of the semiconductor detector compared to other methods. In addition, the analysis system in this study, which was configured by a combination of Monte Carlo simulation and the Hecht model, is very reliable at reconstructing the actual spectrum to study the characteristics of the CZT detectors.

\section{ACKNOWLEDGMENTS}

This study was supported by the Korea Atomic Energy Research Institute (M20704000003-07M040000310), the Ministry of Knowledge Economy (2008-PEP-HM-E-06-0000), and the Innovative Technology Center for Radiation Safety.

\section{REFERENCES}

[ 1 ] J. E. Toney, R. B. James, J. Butler, and et al., Cadmium Zinc Telluride Charged Particle Nuclear Detectors, SAND978216, Sandia National Laboratories, (1997).

[2] Hadong Kim, Leonard Cirignano, Kanai Shah, Michael Squillante, and Philip Wong, "Investigation of the Energy Resolution and Charge Collection Efficiency of $\mathrm{Cd}(\mathrm{Zn}) \mathrm{Te}$ Detectors with Three Electrodes," IEEE Transactions on Nuclear Science, 51[3], 1229-1234 (2004).

[3 ] B. A. Brunett, J. M. Van Scyoc, N. R. Hilton, J. C. Lund, and R. B. James, "The Performance Effects of Crystal Boundaries in Cadmium Zinc Telluride Radiation Spectrometers," IEEE Transactions on Nuclear Science, 47[4], 1353-1359 (2000). 
[4] K. S. Shah, J. C. Lund, and F. Olschner, "Charge Collection Efficiency in a Semiconductor Radiation Detector with a Non-constant Electric Field," IEEE Transactions on Nuclear Science, 37[2], 183-186 (1990).

[ 5 ] T. E. Schlesinger, J. E. Toney, H. Yoon, E. Y. Lee, B. A. Brunett, L. Franks, and R. B. James, "Cadmium Zinc Telluride and Its Use as a Nuclear Radiation Detector Material," Materials Science and Engineering, 32, 103189 (2001).

[6] A. K. Khusainov, A. L. Dudin, A. G. Ilves, V. F. Morozov, A. K. Pusotovoit, and R. D. Arlt, "High Performance P-i-n CdTe and CdZnTe Detectors," Nuclear Instruments and Methods in Physics Research A, 428, 58-65 (1999).

[ 7 ] M. Jung, J. Morel, P. Fougeres, M. Hage-Ali, and P. Siffert, "A New Method for Evaluation of Transport Properties in CdTe and CZT Detectors," Nuclear Instruments and Methods in Physics Research A, 428, $45-57$ (1999).

[ 8 ] Y. Nemirovsky, A. Ruzin, G. Asa, and J. Gorelik, "Study of the Charge Collection Efficiency of CdZnTe Radiation Detectors," Journal of Electronic Materials, 25 1221-1231 (1996).

[9] J. E. Toney, T. E. Schlesinger, and R. B. James, "Modeling and Simulation of Uniformity Effects in $\mathrm{Cd} 1-_{x} \mathrm{Zn}_{\mathrm{x}} \mathrm{Te}$ Gamma-ray Spectrometers," IEEE Transactions on Nuclear Science, 45, 105-113 (1998).

[10] M. C. Veale, P. J. Sellin, A. Lohstroh, A. W. Davies, J.
Parkin, and P. Seller, "X-ray Spectroscopy and Charge Transport Properties of CdZnTe Grown by the Vertical Bridgman Method," Nuclear Instruments and Methods in Physics Research A, 576, 90-94 (2007).

[11] Se-Hwan Park, Yong-Kyun Kim, Sung-Dae Jeon, JangHo Ha, and Duk-Geun Hong, "Mean Free Paths of Charge Carriers in CZT Crystal," Nuclear Instruments and Methods in Physics Research A, 579, 130-133 (2007).

[12] D. Pelowitz (Ed.), "MCNPX User's Manual Version 2.5.0," LA-CP-05-0369, Los Alamos National Laboratory, (2005).

[13] M. D. Reed, Cs. Szeles, and S. E. Cameron, "Computational Modeling of Heat Transport in a Multi-zone High-pressure Vertical Electro-dynamic Gradient CdZnTe Furnace," Journal of Crystal Growth, 289, 494-501 (2006).

[14] K. Hecht, "Zum Mechanismus des lichtelekrischen Primastomes in isolierenden Kristallen," Zeits. Phys., 77, 235- (1932).

[15] Goro Sato, and et al., "Properties of CdZnTe Detectors in the burst alert telescope (BAT) Array," Proceedings of SPIE-International Society for Optical Engineering, 5198, 209-216 (2004).

[16] eV Products, http://www.evproducts.com/.

[17] Goro Sato, Tadayuki Takahashi, and et al., "Characterization of CdTe/CdZnTe Detectors," IEEE Transactions on Nuclear Science, 49[3], 1258-1263 (2002). 